\section{Hartmut Hampl}

DADE BEHRING MARBURG GMBH, Infectious Disease Diagnostics, Marburg, Germany

\section{Introduction:}

\section{Development of HIV Testing}

The development and continuous improvement of diagnostic assay systems for the detection of HIV infections belong to the most challenging tasks for firms working in the field of serodiagnosis of microbial and viral diseases.

Since 1982, soon after it became apparent that HIV may be transmitted via blood transfusion, programs for identifying donors at high-risk were installed in most countries in order to prevent transmission of the disease. Such programs included mandatory testing for surrogate markers such as Hepatitis B core antibodies. A few years later, in 1985, the first antiHIV screening assays were introduced in donor screening and, as a consequence, the risk of transfusion associated HIV infections was significantly reduced. Since the first antibody screening assays became available a continuous improvement of their overall performance, in detail, specificity and sensitivity was achieved up to now. This was possible due to the availability of defined antigens, either recombinant or synthetic, new assay formats and of course the ongoing research in the field of HIV. Consequently in 1989 simultaneous screening for antibodies against HIV-1 and HIV-2 replaced the classical viral antigen based anti-HIV-1 assays.

In the following years, the main efforts of the diagnostic industry were focused on the reduction of the so-called 'window period' by detecting all different subclasses of immunoglobu- lins and designing HIV-specific antigens in a way to detect low-affinity antibodies reminiscent of the early phase of HIV infection. A milestone of such assays was Behring's Enzygnost Anti HIV-1/2 Plus, since this assay was the first which combined the improvement of sensitivity in early seroconversion with the detection of HIV-O by including a specific HIV$\mathrm{O}$ antigen in the test procedure. Another step into this direction was the introduction of HIV P24 antigen screening of blood donors in North America at the beginning of 1996.

Based on the knowledge that the 'window period' may be shortened by P24 antigen testing, Behring now intends to introduce a combined assay for the simultaneous detection of HIV P24 antigen and HIV antibodies for blood donor screening. As expected, preliminary data of this combined assay on seroconversion panels account for the significant rise in sensitivity. In addition, an outstanding performance for specificity is achieved with this combination assay.

Molecular techniques such as DNA probing gain importance in microbial and viral diagnosis. Another milestone may be expected when this technology becomes available for routine testing in transfusion medicine. At the time of implementation, hopefully in the near future, another improvement in the early seroconversion detection of HIV infection will be achieved.

\begin{tabular}{ll}
\hline KARGER & (c) 1998 S. Karger GmbH, Freiburg \\
& Fax (07 61) 4520714 \\
& www.karger.com
\end{tabular}

